\title{
Le rôle de la dépendance externe et des structures sociales dans l'économie frumentaire du Canada et de l'Argentine (1880-1930)
}

\author{
Marc-A. Blain
}

Volume 26, numéro 2, septembre 1972

URI : https://id.erudit.org/iderudit/303175ar

DOI : https://doi.org/10.7202/303175ar

Aller au sommaire du numéro

Éditeur(s)

Institut d'histoire de l'Amérique française

ISSN

0035-2357 (imprimé)

1492-1383 (numérique)

Découvrir la revue

Citer cet article

Blain, M.-A. (1972). Le rôle de la dépendance externe et des structures sociales dans l'économie frumentaire du Canada et de l'Argentine (1880-1930). Revue d'histoire de l'Amérique française, 26(2), 239-270.

https://doi.org/10.7202/303175ar d'utilisation que vous pouvez consulter en ligne. 


\title{
LE ROLE DE LA DÉPENDANCE EXTERNE ET DES STRUCTURES SOCIALES DANS L'ÉCONOMIE FRUMENTAIRE DU CANADA ET DE L'ARGENTINE (1880-1930)*
}

\author{
Marc-A. Blain \\ Département d'histoire \\ Université du Québec \\ à Montréal
}

\begin{abstract}
Au cours des 50 années à la charnière du siècle, principalement pendant les trois premières décennies du $20^{\circ}$, le Canada et l'Argentine ont constitué aux extrémités du continent américain des pôles d'attraction de première importance pour l'Europe, alors encore en mesure de déterminer le rythme et le style de l'évolution de la civilisation. Après les Etats-Unis et avec l'Australie dans une moindre mesure, ces deux nations se révélaient l'archétype des pays neufs où tout était à faire, mais où tout s'annonçait possible à l'intérieur de potentialités si vastes qu'on les imaginait volontiers illimitées. Comme les deux autres contrées, elles ont peu à peu précisé leur rôle de régions-frontières $\mathrm{du}$ Vieux Continent, d'arrière-pays vides et riches que le succès trop rapide de la révolution industrielle poussait à mettre en valeur intensément afin de soulager quelque peu l'Europe de ses trop-pleins et de ses carences.

C'est avec plus d'un demi-siècle de retard sur les EtatsUnis que l'Argentine et le Canada ont amorcé leur boom de croissance quant aux exportations agricoles massives. Celle-là précède son concurrent du nord de deux décennies; mais malgré quelques nuances on peut à bon droit parler de phénomènes synchrones quand il s'agit de l'économie du blé du Canada et de l'Argentine des années 1880-1930. Ceux-ci en effet se rangent

* Cette modeste étude d'histoire comparée repose, je le reconnais, sur une documentation inégale. Les sources primaires argentines me sont beaucoup plus familières que les canadiennes. Le lecteur intéressé à approfondir l'histoire céréalière de La Plata pourra consulter, entre autres, ma thèse non encore publiée : L'économie céalière et son influence sur la croissance économique de l'Argentine, 1870-1930 (Caen : Faculté des lettres et. sciences humaines, mai 1970), 516 p., bibliographie, cartes, graphiques, tableaux.
\end{abstract}

[239]

RHAF, vol. 26, no 2 (septembre 1972) 
dans un contexte international tout à fait identique et répondent aux mêmes forces profondes d'une façon étonnamment semblable au premier regard. Jusqu'au volume de leur population qui reste très voisin: 4-5 millions d'habitants vers 1895 et $10-11$ millions en $1930 .{ }^{1}$ Les similitudes entre les deux économies frumentaires sont nombreuses et peuvent pousser l'observateur trop pressé à des conclusions dangereuses; car derrière la façade des ressemblances se dissimulent de profondes divergences qui dépassent largement le simple mode de fonctionnement pour toucher aux structures mêmes des sociétés concernées. L'étude des points de convergence nous arrêtera vite au niveau des résultats du processus d'économie frumentaire d'exportation; au-delà de cette frontière, l'examen des modalités et des lignes de force amène le chercheur à des constatations assez éloignées de la belle concordance du début. Celle-ci vaut néanmoins l'effort d'un examen rapide ne serait-ce que pour éclairer d'un jour plus vif le reste de l'étude. Au bout du compte - c'est l'essentiel de ce travail - on verra se profiler deux sociétés fondamentalement différentes, mais capables apparemment d'engendrer des phénomènes d'intensité comparable à l'intérieur d'un cadre mondial analogue (voir le tableau 1).

\section{1 - ANALOGIES QUANTITATIVES}

Avec la croissance constante du volume de l'offre mondiale de blé à partir des années 1880, mise à part la baisse conjoncturelle de 1914-1919, le mouvement vers une cristallisation du marché s'est accentué sans cesse au profit d'une dizaine d'approvisionneurs de plus en plus importants. Vers 1880, neuf exportateurs seulement occupaient environ $70 \%$ du marché mondial (voir le tableau 2); à la fin des années 1920, ils comptaient pour $94 \%$. Pendant ces soixante années, les Européens de l'Est glissèrent de $35 \%$ du marché en 1900-1913 à 5\% en 1925-1929. De leur côté, les quatre pays neufs (Etats-Unis, Canada, Argentine et Australie) sont passés de $40 \%$ des exportations mondiales en 1909-1913 à 88\% en 1925-1929.

Avant 1900, deux énormes approvisionneurs se partageaient environ $45 \%$ des exportations mondiales de froment. La Russie et les Etats-Unis expédiaient chacun de un à trois millions de tonnes de blé annuellement, devançant ainsi d'assez loin tous

${ }^{1}$ M. C. Urquhart et K. A. H. Buckley, Historical Statistics of Canada (Toronto, 1965), 14; República argentina, Dirección general de estadística, Anuario del comercio exterior de la República argentina (Buenos Aires, 1935), 8 et 9 . 
Tableau 1

LE BLE AU CANADA ET EN ARGENTINE

Milliers d'hectares et de tonnes métriques, kilos/ha.

\begin{tabular}{|c|c|c|c|c|c|c|c|c|c|c|c|c|c|}
\hline & \multicolumn{2}{|c|}{ surf. semées } & \multicolumn{2}{|c|}{ production } & & \multicolumn{2}{|c|}{ rendement } & \multicolumn{2}{|c|}{ exportation } & \multicolumn{2}{|c|}{$\%$ de prod. } & \multicolumn{2}{|c|}{ export.farine } \\
\hline & $\underset{1}{\text { Arg. }}$ & $\underset{2}{\mathrm{Can} .}$ & $\underset{3}{\text { Arg. }}$ & $\underset{4}{\mathrm{Can}}$ & & $\underset{5}{\operatorname{Arg} .}$ & $\underset{6}{\text { Can. }}$ & $\underset{7}{\text { Arg. }}$ & $\underset{8}{\text { Can. }}$ & $\underset{9}{\operatorname{Arg} .}$ & $\begin{array}{c}\text { Can. } \\
10\end{array}$ & $\underset{11}{\text { Arg. }}$ & $\begin{array}{c}\text { Can. } \\
12\end{array}$ \\
\hline $885-89$ & 800 & 1000 & 500 & 1000 & $1884-88$ & - & - & 128 & 78 & 25 & 8 & 6 & 28 \\
\hline $1889-94$ & 1400 & 1200 & 1300 & 1100 & $1889-93$ & - & 一 & 445 & 114 & 34 & 10 & 6 & 24 \\
\hline $1894-99$ & 2500 & 1500 & 1600 & 1400 & $1894-98$ & - & - & 787 & 299 & 49 & 21 & 44 & 45 \\
\hline $1899-04$ & 3500 & 1700 & 2500 & 2100 & $1899-03$ & $685^{1}$ & $1456^{1}$ & 1375 & 557 & 55 & 26 & 59 & 97 \\
\hline 1904-09 & 5600 & 2300 & 4300 & 2800 & $1904-08$ & 770 & 1246 & 2748 & 944 & 64 & 34 & 124 & 139 \\
\hline $1909-14$ & 6400 & 4000 & 4000 & 5400 & $1909-13$ & 616 & 1326 & 2425 & 2021 & 61 & 37 & 121 & 328 \\
\hline $1914-19$ & 6600 & 5800 & 4600 & 6800 & $1914-19$ & 698 & 1106 & 2168 & 3101 & 47 & 46 & 157 & 697 \\
\hline $1919-24$ & 6400 & 8400 & 5500 & 8700 & $1920-24$ & 850 & 1060 & 3724 & 5098 & 67 & 58 & 123 & 785 \\
\hline $1924-29$ & 8000 & 9000 & 6800 & 11700 & $1925-29$ & 830 & 1262 & 4252 & 7097 & 63 & 61 & 151 & 895 \\
\hline
\end{tabular}

Tous ces chiffres représentent des moyennes de 5 ans sauf 1914-19 (6 ans) et 1885-89 (4 ans).

1 1901-1903.

Sources : colonnes 1 à 4 : d'après Malenbaum, Wilfred, The World Wheat Economy, 1885-1939. Cambridge, 1953. colonnes 5 à 6 : d'après ibid. et Institut international d'agriculture de Rome, Annuaire international de statistique agricole. Rome, 1909-1930.

colonnes 8 et 12 : Institut international d'agriculture de Rome, op. cit., et Broomhall, George J. S. Corn Trade Yearbook; International Grain Trade Statistics. Liverpool, 1895-1903. colonnes 7 et 11 : Scobie, James R. Revolution on the Pampas. Austin, 1964. p. 170; Aparicio, Francisco de et al. Anuario geográfico argentino. Buenos Aires, 1941. p. 379. 
leurs concurrents. ${ }^{2}$ D'autre part, aucun autre pays n'exportait plus de 950000 tonnes par année. Au cours de la décennie précédant la guerre, les Etats-Unis ne régnaient plus seuls: l'Argentine s'était installée elle aussi au palier du million de tonnes et plus (elle dépassait même les Etats-Unis en 1904-1913) et elle a été bientôt imitée par l'Inde, la Roumanie, l'Australie et surtout par le Canada. Pendant les années 1914-1929, le volume des exportations mondiales de froment s'est stabilisé aux environs de 17 millions de tonnes. Les pays d'outre-mer se sont constitués en un groupe relativement homogène de très gros pourvoyeurs de blé. Le Canada, les Etats-Unis et l'Argentine ont chacun exporté plus de 3 millions de tonnes par an pendant

Tableau 2

LES EXPORTATIONS DE BLÉ EN GRAIN (pourcentage par rapport aux exportations mondiales) (moyennes quinquennales)

\begin{tabular}{|c|c|c|c|c|c|c|c|c|c|c|}
\hline & 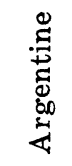 & 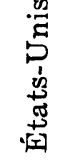 & 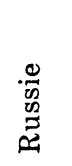 & 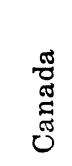 & 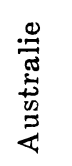 & $\underset{\Xi}{\stackrel{\Xi}{\Xi}}$ & 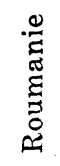 & 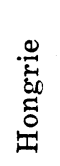 & 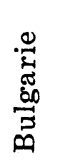 & 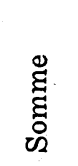 \\
\hline $1884-88$ & 1,3 & 21,8 & 24,3 & 0,7 & 2,5 & 10,2 & 4,8 & 2,4 & 2,3 & 70,3 \\
\hline 1899-03 & 11,5 & 26,9 & 22,0 & 4,7 & 2,1 & 4,6 & 5,4 & 1,0 & 1,6 & 79,8 \\
\hline $1904-08$ & 19,6 & 10,9 & 24,0 & 6,7 & 5,1 & 7,4 & 8,6 & 3,6 & 2,4 & 88,3 \\
\hline $1909-13$ & 13,8 & 8,3 & 24,2 & 11,5 & 6,5 & 7,5 & 7,6 & 2,3 & 1,2 & 82,9 \\
\hline $1914-19^{1}$ & 17,3 & 32,6 & 3,8 & 24,8 & 9,1 & 5,5 & 1,3 & 0,2 & 0,3 & 94,9 \\
\hline $1920-24$ & 21,9 & 29,7 & 0,7 & 29,9 & 10,2 & 2,3 & 0,3 & 0,3 & 0,3 & 95,6 \\
\hline $1925-29$ & 22,7 & 16,8 & 2,4 & 37,8 & 10,6 & 1,3 & 0,6 & 1,8 & 0,1 & 94,1 \\
\hline
\end{tabular}

1 Moyenne de six ans.

Sources : voir la note 2.

2 Ces données sont celles qui ont permis de dresser le tableau 2 et la série d'indices qui suivent. Aux sources citées au tableau 1, il faut ajouter: Paul van Hissenhoven, Le mouvement des grains dans le monde (Bruxelles, 1938), passim; Vladimir P. Timoshenko, Wheat Prices and the World Wheat Market (New York, 1927), passim; Rollin E. Smith, Wheat Fields and Markets of the World (Saint Louis, 1908), passim; Stanford University, Food Research Institute, Wheat Studies (1924-1944), passim; E. Dunsdorfs, The Australian Wheat Growing Industry (Londres, 1956), 476; M. W. Menzies, The Canadian Wheat Board and the International Wheat Trade, (thèse) (Londres, 1956), passim. 
cette période et l'Australie 2 millions. A eux seuls, les quatre grands fournissaient au monde de $78 \%$ à $80 \%$ du blé acheté à l'étranger.

En examinant globalement la période de 1875 à 1929 on voit que les trois pays qui comportaient le plus de similarités (une faible population, de vastes territoires inexploités, une économie d'exportation, etc...) ont montré également un certain parallélisme dans leur trend d'exportation de blé. L'Argentine, le Canada et l'Australie furent les seuls à connaître une croissance aussi rapide, sans comparaison avec celle de leurs concurrents. Entre 1884-1888 et 1925-1929, les indices du volume d'exportation ont bondi respectivement de 5 à 175 , de 4 à 351 et de 21 à 174. Si on coupe la période en 1904-1908 on voit que les tendances de l'Argentine avant et après ce lustre ont suivi de près la propension générale; ainsi les exportations de ce pays ont augmenté selon un rythme assez constant malgré la stagnation de 1904-1908 à 1914-1919. Dans la première période, le Canada a accru ses ventes de blé à un rythme plutôt lent, se laissant devancer largement par son concurrent argentin jusqu'en 1910, mais les a augmentées à une vitesse extraordinaire après 1904-1908, jusqu'à supplanter assez décisivement l'Argentine au cours des années 1920. L'Australie n'a amorcé sa croissance qu'en 1894-1898 et a suivi ses concurrents avec un net retard.

Vus de plus haut encore, les exportateurs se sont répartis en deux groupes: les progressifs et les régressifs, les pays neufs et les vieux pays, les pays d'outre-mer et les Européens. Il est inutile d'insister sur le rôle de catalyseur que le premier conflit mondial a joué dans cette polarisation. Mais à long terme, les vieux producteurs comme l'Inde et les pays du Danube étaient battus d'avance. Leur situation géohistorique leur a imposé des limitations beaucoup plus sévères que celles dont ont souffert les pays neufs. Leurs populations denses, en augmentant la demande interne, ont forcément diminué la capacité exportatrice et l'épuisement des terres disponibles a mis un frein à la croissance horizontale de l'agriculture alors que leurs modes d'exploitation ne leur permettaient pas de compenser par une croissance verticale. Les pays neufs au contraire ont pu surmonter l'obstacle de l'éloignement des centres importateurs - surtout après la diminution du fret maritime à la fin du $19^{e}$ siècle et leurs faibles rendements par une technologie progressive qui a permis d'effacer le désavantage d'une population agricole relativement réduite et qui a rendu possible une croissance horizontale quasi-illimitée. De ce bref examen des structures du 
marché d'exportation de blé apparaît l'importance capitale des pays d'outre-mer et en particulier l'extraordinaire dynamisme du Canada et de l'Argentine (voir les tableaux 1 et 2 ). Ces derniers n'occupaient au cours des années 1880 qu'une place obscure (environ $2 \%$ des exportations mondiales) ; mais ils fournissaient au monde $60 \%$ du froment exporté à la fin des années 1920. Ces bonds en avant spectaculaires méritent à eux seuls d'être soulignés tant par leur importance quantitative que par leur parallélisme. Ils constituent en tout cas le plus visible des facteurs de similitude entre les économies frumentaires argentine et canadienne.

Un second point de convergence réside dans la très forte dépendance des économies du blé argentine et canadienne à l'égard des marchés d'exportation. Peu peuplés et produisant du blé à la cadence que l'on sait, ces pays ont vendu à l'étranger une forte part de leur production (voir le tableau 1). Dès 1898, la République de La Plata exportait plus de la moitié de son blé et environ les deux-tiers après 1905, sauf pendant la guerre. Après 1905, le Canada a lui aussi expédié une proportion appréciable de son froment hors de ses frontières: le tiers jusqu'à la guerre et près des deux-tiers au cours des années 1920, alors qu'à ce point de vue il avait presque rejoint l'Argentine. Ainsi, dans les deux cas et surtout à partir de 1914, quand les exportations de ces deux nations ont atteint des volumes gigantesques, les économies du blé se sont alignées surtout sur les marchés extérieurs. De ce fait, elles ont de toute évidence noué avec des économies étrangères des liens serrés de dépendance ou, à tout le moins, de vulnérabilité. Les secteurs frumentaires jouant à l'intérieur des économies nationales respectives un rôle de première importance, cette situation comportait tous les risques de prospérité en phase conjoncturelle mondiale ascendante et de crise en période de dépression. Les années 1925-1935, dans l'un et l'autre pays, et en sens inverse, ont éloquemment souligné la gravité de ces implications. Autant la prospérité fébrile de la fin des années 1920 a stimulé les ventes de céréales, autant la débâcle des années 1930-1935 les a fait s'effondrer. Pendant la période qui retient notre attention, les acheteurs de blé argentin aussi bien que canadien se sont situés en Europe de l'Ouest. Depuis la révolution industrielle, cette région est restée la principale zone de la planète où la production de blé ne suffisait pas à la consommation. Entre 1894 et 1913 , elle accaparait $87 \%$ des exportations mondiales de blé. ${ }^{3}$ De cette date à 1929 , les huit plus importants importateurs européens exigeaient à eux seuls de

3 Timoshenko, op. cit., 6. 
$72 \%$ à $76 \%$ du blé vendu à l'étranger. Parmi eux, la GrandeBretagne occupait une place à ce point prépondérante qu'elle constituait de loin le plus gros acheteur mondial de blé. Sa part du volume mondial des importations a oscillé entre $28 \%$ et $35 \%$ de 1885 à 1930. Les autres ventes de froment se dirigeaient surtout vers les Pays-Bas, la Belgique, l'Allemagne et l'Italie qui réunis recevaient à peine plus que l'Angleterre. ${ }^{4}$ Ainsi, la structure du marché d'importation apparaissait au moins aussi concentrée que celle de l'exportation. Pour le Canada et l'Argentine, cependant, c'est dans une proportion beaucoup plus forte que la part britannique du marché mondial que les ventes de blé à ce pays se sont effectuées. Si on peut estimer grossièrement que le premier acheminait environ la moitié de son blé vers l'Angleterre avant 1914, on calcule aussi que cette proportion s'élevait à 78-80\% à partir de 1914-1919. La République sud-américaine de son côté vendait aux Anglais environ $40 \%$ de son froment avant la guerre et près de $25 \%$ à la fin des années $19200^{5}$ L'importance primordiale du marché britannique apparaît donc clairement, surtout dans le cas canadien. Voilà une troisième similitude digne de mention.

Il n'entre pas dans mon propos d'en traiter de façon détaillée, mais il importe de remarquer au passage que la dépendance des économies frumentaires argentine et canadienne envers le client britannique ne constituait pas un phénomène isolé. Ce sont les bateaux anglais qui transportaient l'essentiel des cargaisons de blé, qu'ils fussent des tramps ou des bateaux des lignes régulières comme la Royal Mail Steam Packet, la Lamport and Holt Line, la Blue Star Line ou la Nelson Line. ${ }^{8}$ Parmi les grandes maisons d'exportation de blé figuraient dans l'un et l'autre pays de nombreuses et puissantes sociétés anglaises, suc-

${ }^{4}$ Mêmes sources que pour la note 2.

5 D. A. MacGibbon, The Canadian Grain Trade (Toronto, 1932), 438; Paul de Hevesy, World Wheat Planning and Economic Planning in General (Londres, 1940), 752; Werner Schlote, British Overseas Trade from 1870 to the 1930's (Oxford, 1952), 164-165; Grande-Bretagne, Board of Trade, Annual Statement of the Trade of the United Kingdom with Foreign Countries and British Possessions for the Year [1870-1930]; República argentina, Dirección general de estadística, Anuario del comercio exterior de la República argentina [1885-1909 et 1925-1929]; Miguel A. Cárcano et Raúl Prebisch, Anuario de la Sociedad rural argentina (Buenos Aires, $1928), 140,175$ et 198.

6 William P. Rutter, Wheat Growing in Canada, United States and Argentina (Londres, 1911), 187-188 et 260; James D. Whelpley, Trade Development in Argentina (Washington, 1911), 24; William Goodwin, Wheat Growing in the Argentina Republic (Liverpool, 1895), 46; The Economist, "Argentina Supplement", (14 déc. 1929) : passim. 
cursales d'entreprises d'importation sur le continent européen. D'une manière générale, l'Argentine et le Canada entretenaient, surtout avant 1914, des relations commerciales très étroites et même prépondérantes avec la Grande-Bretagne; et sur le plan financier, ils servaient de terrain privilégié d'investissement à cette métropole du capitalisme européen. En 1913, par exemple, on comptait 515 millions de livres investies par l'Angleterre au Canada et Terre-Neuve et pas moins de 320 millions en Argentine; dans ce dernier cas, c'était $35 \%$ de plus que la somme des investissements anglais dans toute l'Europe et presque autant que les capitaux exportés en Inde-Ceylan ou en Afrique du Sud. En 1929, les avoirs britanniques se chiffraient à environ 500 millions de livres en Argentine, plus que les 440 millions recensés au Canada. ${ }^{7}$ Dans ces conditions, on perçoit aisément que la dépendance des économies frumentaires canadienne et argentine s'intégrait dans un ensemble de relations néo-métropoles-colonies beaucoup plus tentaculaires qu'il paraît quand on l'étudie isolément. Encore une fois la République platéenne et le Dominion du Nord se retrouvaient dans la même galère.

\section{2 - SIMILITUDES DE CONJONCTURES}

Pour compléter l'énoncé des éléments d'analogie entre les économies du blé argentine et canadienne, il faut ouvrir un second volet, celui de leur intégration presque simultanée dans le grand mouvement conjoncturel de la période triomphale de la révolution industrielle, époque que plusieurs préfèrent appeler l'ère du capitalisme monopoliste. A cet égard aussi les deux pays ont suivi des chemins parallèles qui les ont conduits à des résultats très voisins en apparence.

Pour nécessaire qu'elle ait été, la stabilisation politique du Canada et de l'Argentine vers 1865-1880 n'aurait certes pas suffi à donner à l'économie frumentaire un rythme de croissance de l'ampleur de celui que j'ai décrit. Mais ce mouvement a coïncidé avec la diffusion des conquêtes de la révolution industrielle de l'Europe et il s'en est trouvé fortement accéléré. A la fin du $19^{\circ}$ siècle, l'économie mondiale a traversé une phase d'expansion d'une rapidité et d'une ampleur encore jamais vues. Le bouleversement économique de l'Europe s'est traduit à l'extérieur par la diffusion d'une technologie révolutionnaire dans le domaine des transports, par une augmentation fabuleuse du

7Herbert Feis, Europe the World's Banker, 1870-1914 (New Haven, 1930), 16 et 23; The Economist, “Argentine Supplement", (14 déc. 1929): 1 . 
commerce international, par des flots d'immigrants et de capitaux. Vue sous cet angle; l'expansion des secteurs céréaliers de nos deux pays ne constituait qu'un épisode du grand essor européen. ${ }^{8}$

Dans ces importantes transformations, les innovations techniques ont certainement constitué le plus puissant des catalyseurs. C'est dans la mesure où les problèmes de transport entre autres ont trouvé une solution économique que les économies régionales ont pu s'intégrer les unes aux autres et que des marchés réciproques ont pu se créer entre des pays situés aux antipodes de la planète. A cet égard, les chemins de fer et les bateaux à vapeur ont joué un rôle de tout premier plan. Entre 1876 et 1896 seulement, la flotte commerciale mondiale est passée de 5,7 millions de tonnes (à vapeur) et 17 millions de tonnes (à voile) à 15,5 millions et à 9,1 millions respectivement. De 1879 à 1900, le prix du transport intercontinental a diminué radicalement, de l'ordre de 4 à 1 dans le cas du transport du blé entre New York et Liverpool. Avec l'utilisation grandissante du chemin de fer (le monde en comptait $367000 \mathrm{~km}$. en 1880 et $740000 \mathrm{~km}$. en 1898), le trafic global de marchandises et de passagers a fait un prodigieux bond en avant, surtout si on considère le prix que coûtait le transport terrestre avant le chemin de fer. ${ }^{9}$ De fait, le réseau de chemins de fer canadien comptait 7200 milles en 1880, 16000 milles en 1895, 20600 en 1905,40600 en 1910 et 55800 en 1929, la période d'expansion intense se situant avant 1920. En Argentine, le réseau est passé aux mêmes dates de $2500 \mathrm{~km}$. à 14100 , à 33500 puis à 38863 ; là aussi l'essentiel avait été fait avant la guerre ${ }^{10}$ (voir les graphiques 3 et 4 ). Les progrès de la technique ne s'étant pas limités aux moyens de transport, tous les secteurs de la vie économique ont été transformés par des innovations accélérées. Mentionnons seulement la mise au point de machines agricoles de plus en plus efficaces sans lesquelles l'agriculture argentine et canadienne ne serait pas sortie de l'époque coloniale. En somme, dans la seconde moitié du $19^{\mathrm{e}}$ siècle, les conditions de mise en valeur des richesses naturelles se sont complètement modifiées. Les hommes ont acquis en peu de temps une extra-

${ }^{8}$ Aldo Ferrer, La economía argentina (Mexico, 1963), 91.

9 Ferrer, op. cit., passim, 91-95; Malenbaum, op. cit., 40; Timoshenko, op. cit., 18; Ricardo M. Ortiz, Historia económica de la Argentina, 1850-1930 (Buenos Aires, 1955), I: 169-170 et 119-120; James R. Scobie, Argentina (New York, 1964), 109.

10 Urquhart et Buckley, op. cit., 528 et 532; República argentina, Ministerio de agricultura, Anuario agropecuario (1932): 17 et (1935): passim. 


\section{8}

graphique 3

\section{Argentine}

$10 \mathrm{M}$

IM

100000

10000

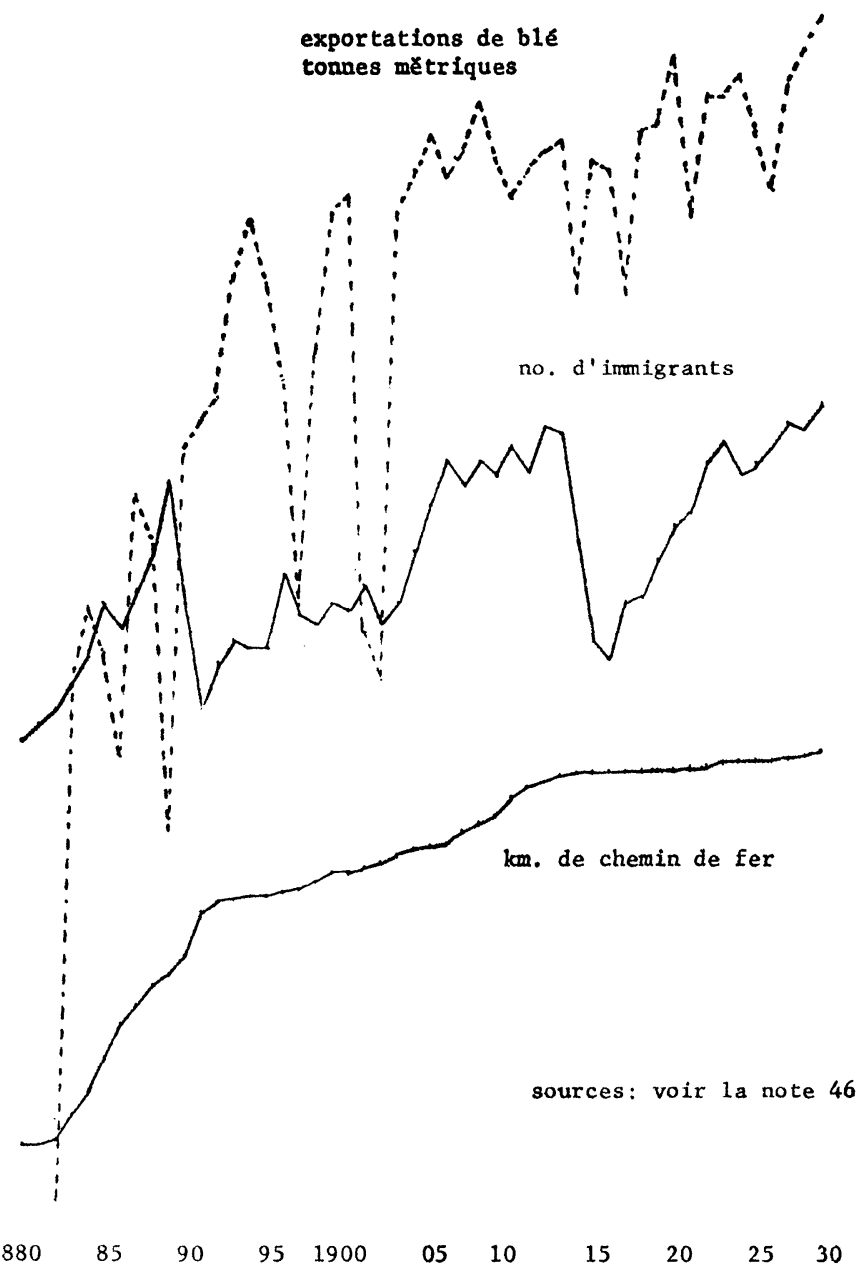

1000 


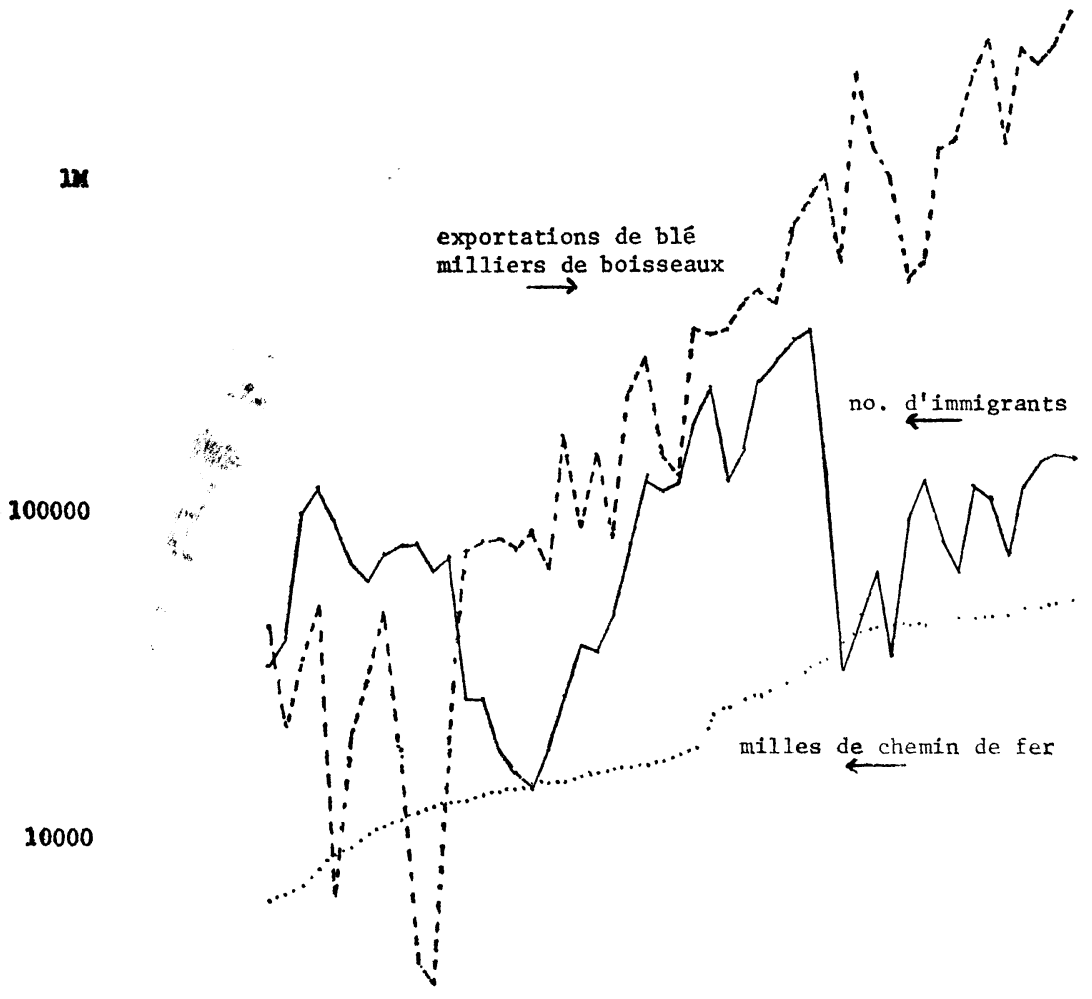

sources: voir la note 47 
ordinaire capacité de produire et d'échanger. Ce qui était impensable en 1850 était devenu affaire de routine en 1895.

Pour réaliser leurs grandes possibilités économiques, l'Argentine et le Canada avaient un immense besoin de population, autant pour se créer une main-d'œuvre que pour se constituer un marché interne de consommation. La croissance végétative ne suffisait manifestement pas à satisfaire cet appétit. Dans les conditions de 1860-1870, et à plus forte raison avant, le souspeuplement représentait un véritable frein à la croissance de l'économie. Cette vérité s'imposait avec une évidence telle que les gouvernants argentins avaient inscrit dans la constitution du pays (article 25) le devoir des pouvoirs publics de veiller à encourager l'immigration européenne et qu'au Canada les gouvernements pratiquaient depuis plusieurs décennies déjà une politique active de recrutement des immigrants. Or, dans la seconde moitié du siècle, l'Europe a fourni aux pays neufs des masses migratoires très importantes que les possibilités économiques et sociales de ces régions attiraient et que la révolution industrielle poussait à chercher une amélioration de leur sort ailleurs que dans leur continent d'origine alors en forte poussée démographique. Au cours des années 1830, environ 100000 personnes quittaient le Vieux Continent chaque année; mais pendant la décennie 1870,310000 personnes se dirigeaient annuellement vers l $\in \mathbf{S}$ Etats-Unis, le Canada, le Brésil et l'Argentine et ces chiffres sont de 660000 de 1880 à 1890 et de 570000 au cours des dix dernières années du siècle. ${ }^{11}$ L'Europe jouait de cette manière encore un rôle capital dans le développement des pays du Nouveau-Monde. De 1870 à 1930, l'Argentine a accueilli des millions d'immigrants dont 3430000 se sont fixés à demeure. ${ }^{12}$ De ceux-là environ $80 \%$ ont élu domicile dans la région pampéenne - la plupart dans les villes il est vrai - apportant ainsi la main-d'œuvre indispensable à l'industrie du blé toujours avide de bras malgré la mécanisation. Le Canada a connu un mouvement du même genre: de 1900 à 1914 seulement il a accueilli 2906000 immigrants dont 1225000 se sont dirigés dans les plaines de l'Ouest. ${ }^{13}$ Devant ces chiffres et à l'examen des graphiques 3 et 4 qui montrent bien, surtout dans le cas argentin, le parallélisme des fluctuations entre le niveau d'activité de l'économie du blé (exportations) et le volume de l'immigration,

11 Ferrer, op. cit., 97; Walter F. Willcox, ed., International Migrations (New York, 1929), I: 172.

12 República argentina, Dirección general de estadística, El comercio exterior argentino en 1936 y 1935 (Buenos Aires, 1937), 105-107.

13 MacGibbon, op. cit., 41. 
il est impossible de ne pas voir ce qui du reste va de soi; il existe une étroite relation entre ces deux facteurs, auxquels on peut ajouter la construction du chemin de fer.

De la même façon que le succès de la transformation économique de l'Europe a engendré des mouvements d'hommes et de capitaux, les nouvelles structures de l'économie occidentale ont libéré d'énormes quantités de marchandises pour le commerce international. L'Europe industrialisée a commencé à déverser sur le monde des biens manufacturés de plus en plus diversifiés et à réclamer les produits primaires qui lui faisaient défaut. Ainsi s'est dessinée une spécialisation internationale qui a déclenché un essor remarquable des échanges. Désormais capables d'approvisionner massivement les pays neufs en biens d'équipement et de consommation, les Européens ont acheté en échange les aliments et les matières premières que le Nouveau-Monde pouvait produire en surabondance. De 1700 à 1820 , le volume commercial mondial avait triplé; mais de 1870 à 1913 - en deux fois moins de temps donc - il s'est multiplié par cinq. ${ }^{14}$

Aucun de ces facteurs n'a pu se développer seul et ils se sont influencés mutuellement jusqu'à former un tout indissociable. Le progrès de l'économie céréalière de l'Argentine et du Canada dépendait tout à la fois de l'immigration, des investissements, de la technologie et de la stabilisation politique; et l'affaiblissement d'un des éléments entraînait nécessairement un amoindrissement des autres. Or, les morceaux du casse-tête se sont réunis vers 1880 dans le premier cas et 1895 dans le second: à compter de ces dates, le progrès s'est imposé presque de luimême. Pour que cet ensemble de circonstances favorables pût déclencher un processus de croissance appuyé sur l'exportation agricole, il ne manquait plus que le facteur "possibilité-naturelle-deproduction". Les terres vierges, immenses et fertiles, c'est bien ce que les Argentins et les Canadiens possédaient à satiété.

En somme, dans les deux pays la croissance de l'économie du blé a accompagné ou résulté de l'ouverture des "frontières" aux locomotives et aux immigrants, ouverture conditionnée en arrière-plan par les forces et les besoins nouveaux dérivés des changements économiques en Europe (voir les graphiques 3 et 4). La synchronisation et l'ampleur des économies frumentaires argentine et canadienne ne tiennent pas du hasard. Elles découlent pour l'essentiel d'un même grand phénomène sur lequel ni les Argentins ni les Canadiens n'avaient beaucoup de prise, à savoir la révolution industrielle européenne, anglaise avant tout,

14 Ferrer, op. cit., 97-99. 
qui a engendré une demande de produits alimentaires et qui a fourni aux régions périphériques du globe les moyens technologiques et autres d'y répondre. En d'autres mots, les économies frumentaires de ces deux pays furent en grande partie marquées dans leurs fondements mêmes par des réalités étrangères à leurs agrégats économiques respectifs. Ce sont assurément là deux exemples parfaits de ce que les marxistes appellent des économies périphériques.

\section{3 - DISSEMBLANCES DES SYSTEMES DE MANUTENTION}

Dans deux cas comme ceux-ci où les conditionnements profonds s'apparentent étroitement l'un à l'autre, on serait en droit d'attendre des similitudes à d'autres niveaux des économies du blé, dans les modalités et les résultats par exemple. Or on constate avec une certaine surprise que ces deux ensembles examinés de près présentent beaucoup plus de différences que de ressemblances. Une comparaison minutieuse déborderait largement le cadre de cette étude; aussi je me limiterai à mettre en lumière le style et les techniques de mise en marché du blé dans chaque pays. C'est un bon échantillon de la rupture dans la continuité que j'ai décrite jusqu'ici.

Un aspect des économies céréalières des pays neufs a constamment suscité des commentaires et appelé des comparaisons: le développement d'un réseau d'élévateurs à céréales en relation avec la qualité des froments mis en marché, avec les coûts de production et les prix qu'on pouvait en retirer. Dans la documentation argentine en particulier, cette préoccupation apparaît primordiale et permanente, d'autant plus sans doute que ce fier pays souffrait de ne pas disposer de l'équipement dont se prévalait le Canada, un de ses principaux rivaux sur les marchés du blé.

$\mathrm{Au}$ Canada, le blé est toujours passé par les élévateurs, c'est-à-dire qu'il a été commercialisé en vrac et non en sacs, du moins au cours de la période qui m'intéresse ici. La construction d'un réseau d'élévateurs a emboîté le pas au développement de l'Ouest céréalier; il a même précédé la grande période d'exportation massive qui ne s'ouvre qu'avec le $20^{\mathrm{e}}$ siècle puisque le premier élévateur-terminus fut construit à Port Arthur en 1884. ${ }^{15}$ En 1901, au début de son boom d'exportation de blé, le

15 MacGibbon, op. cit., 86. 
Canada disposait déjà d'une capacité de stockage de 252000 tonnes métriques dans les Prairies et de 218000 tonnes dans les ports des Grands Lacs et les ports de mer. Jusqu'en 1929, le rythme d'installation du réseau n'a jamais ralenti: la capacité des Prairies est passée de 2,4 millions de tonnes en 1914 à 5,3 millions en 1929; celles des terminus a suivi la même courbe, de 2,2 millions de tonnes en 1914 à 5,5 millions en $1929 .{ }^{16} \mathrm{Si}$ on considère comme normal qu'un élévateur-terminus manipule pendant un an jusqu'à cinq fois plus de céréales que sa capacité d'entreposage, le réseau canadien offrait toutes les garanties de la suffisance, même si les opérations de manutention se concentraient à $50 \%$ dans les trois mois suivant la récolte.

La mise en place d'un réseau d'élévateurs ne constitue que les fondements de tout un système de mise en marché qui peut dès lors prendre un style tout à fait caractéristique si on le compare à la mise en marché du grain ensaché. L'existence d'une telle infrastructure a permis aux Canadiens de vendre des blés soigneusement pesés, nettoyés, séchés et classifiés selon leur qualité. Ces opérations ont été conduites de façon systématique sous l'œil sévère des inspecteurs du gouvernement. Vers 19091913, $60 \%$ du blé produit dans l'Ouest subissait une inspection; vers 1923-1929, cette proportion s'élevait à $90 \%$. Un tel système où tout le blé passait obligatoirement par l'élévateur et par les opérations qu'on y pratiquait a permis d'atteindre deux résultats très importants. Sur les marchés européens, le blé canadien s'est toujours vendu dans les meilleures conditions possibles: il arrivait propre, sec, pesé et surtout classifié par types. Aussi les importateurs ont-il toujours accepté le blé canadien sur la foi des certificats officiels d'inspection et non sur échantillon comme c'était le cas des froments de presque tous les autres pays. Il en résultait pour le Canada une excellente position commerciale face à ses concurrents et aussi de meilleurs prix pour ses céréales. En second lieu, cette méthode permettait l'émission de certificats de dépôt (cash tickets ou warrants) aux agriculteurs qui pouvaient ainsi toucher dès la livraison de leurs récoltes une partie au moins des recettes ou qui, de cette manière, obtenaient facilement du crédit des banques. ${ }^{17}$ Dans les deux cas, les résultats apparaissaient très avantageux.

16 D'après Urquhart et Buckley, op. cit., 382 et Ministère du commerce du Canada, Annuaire du Canada, 1913: 434-436.

17 MacGibbon, op. cit., 101,112 et 183; Ministère du commerce du Canada, Annuaire du Canada, 1912: 292 et 451; 1935:1030; 1910; 1911; $1913 ; 1924 ; 1925 ; 1929 ; 1931$. 
L'Argentine figurait au rang des producteurs de blé où les opérations de manutention se faisaient surtout selon le système des sacs. A l'intérieur de la pampa, les seuls moyens d'entreposage qui aient réellement existé sont les hangars des gares de chemins de fer qui mettaient les sacs de céréales à l'abri des intempéries dans une proportion importante mais étaient totalement inadaptés au système de mise en marché en vrac. D'ailleurs la généralisation des hangars de gares ne datait que des années 1903-1910, vingt ans après le début de la phase d'exportation massive de blé. Avant le tournant du siècle, ces entrepôts sommaires n'existaient à peu près nulle part sauf dans certaines régions à production agricole particulièrement intense. En 1905, les hangars de la pampa pouvaient contenir 26 millions de sacs (1,7 million de tonnes) ; en 1916 ils avaient augmenté leur capacité à 5 millions de tonnes, c'est-à-dire à plus de $50 \%$ du niveau des exportations. A cette date, le réseau d'entrepôts avait atteint sa maturité et il n'a pas changé jusqu'à 1930. Relativement suffisant pendant les années de production moyenne, il devenait franchement insatisfaisant en période de fortes récoltes, comme pendant les années 1925-1929 ${ }^{18}$.

Vers 1915 l'Argentine ne disposait d'aucun silo de campagne. De 1915 à 1927, dans les cinq provinces pampéennes, des entreprises privées ont bâti 25 élévateurs de campagne d'une capacité globale de stockage d'environ 80000 - 85000 tonnes. Mais en fait, seulement 9 de ces élévateurs (20000 tonnes) travaillaient pour l'exportation; tous les autres ne servaient qu'aux besoins propres des entreprises de meunerie. Par rapport au niveau de production des années 1920, c'était évidemment d'une insignifiance absolue. ${ }^{19}$ Jusque vers 1900 , la situation dans les principaux ports n'était guère prometteuse quant aux possibilités de mise en marché en vrac. Un seul port s'était équipé d'élévateurs d'une capacité globale de 25000 tonnes. C'est entre 1900 et 1914 que l'essentiel du réseau des élévateurs portuaires a vu le jour. En 1915, les trois principaux havres du pays pouvaient accumuler 218000 tonnes, ce qui permettait l'exportation d'une partie des céréales en vrac mais ce qui restait largement en deçà du niveau des exportations. L'Argentine de 1914

18 República argentina, Ministerio de agricultura, Boletín del Ministerio de agricultura, 1905; 172 et passim; id., Valor de la producción nacional (Buenos Aires, 1916), 7; Anales de la Sociedad rural argentina (mars-avril 1905) : 27; Emilio Coni, El mercado ordenado del trigo argentino (Buenos Aires, 1932), 31.

19 The Economist (27 janvier 1923) : 149; República argentina, Ministerio de agricultura, Informe sobre la implantación de un sistema general de elevadores de granos (Buenos Aires, 1928), 35, 71-73 et passim. 
n'avait pas réussi à moderniser ses méthodes de mise en marché des céréales et s'était laissée dépasser par la croissance extraordinaire de ses exportations agricoles. Les embouteillages dans les ports et les retards de livraison survenaient fréquemment. A la veille de la guerre mondiale, au surplus, la grande majorité des élévateurs existants se contentaient de charger les grains en vrac sans les nettoyer ni les classifier, quoique ce fût là une de leurs principales tâches. A la fin des années 1920, la capacité totale des élévateurs portuaires n'était passée qu'à 322000 tonnes, c'est-à-dire $25 \%$ du nécessaire. ${ }^{20}$ En 1903-1904, $70 \%$ du froment arrivait en Europe dans ses sacs originaux et cette proportion a régressé à seulement 50\% au cours des années 1920 . Ainsi le système de mise en marché des céréales en vrac a gagné quelque importance dans les grands ports, il n'a jamais pris pied à l'intérieur du pays. ${ }^{21}$

Les conséquences de l'absence d'un réseau d'élévateurs intégral en Argentine ont lourdement pesé sur l'état final du blé vendu en Europe. Les froments de la pampa se vendaient selon des critères de classification plus que sommaires, l'inspection des sacs étant évidemment une entreprise très difficile. En outre, les opérations de séchage-nettoyage ne pouvaient pas se mener de façon aussi systématique qu'au Canada. Il s'ensuivait forcément que l'acheteur, jamais assuré de la constance de la qualité d'un sac de blé à l'autre, s'accordait toujours une marge de sécurité sur le prix afin de pallier l'irrégularité de la présentation du produit. En fait, contrairement au cas canadien, c'est l'importateur, surtout la London Corn Trade Association, qui établissait lui-même la classification du blé argentin selon la "bonne moyenne de qualité" mensuelle. ${ }^{22}$ Ainsi, le blé de La Plata se trouvait face à son concurrent nord-américain en position de

20 Scobie, Revolution... op. cit., 106-107 et 97; South American Journal (19 mars 1892): 323; René Musset, Le blé dans le monde (Paris, 1923), 164; Laurel Duval, The Production and Handling of Grain in Argentina (Washington, 1915), 295-296; Anales de la Sociedad rural argentina (1905) : 29; República argentina, Ministerio de agricultura, Informe ... op. cit., 77, 79 et passim.

21 Carlos D. Girola, Investigación agrícola en la República argentina (Buenos Aires, 1904), 303; Anales de la Sociedad rural argentina (1904): 338 et 341 ; República argentina, Ministerio de agricultura, Informe ... op. cit., passim.

22 Grande-Bretagne, Chambre des Communes, Accounts and Papers. Diplomatic and Consular Reports (Buenos Aires, 22 janvier 1892), vol. 81 (1892): 15; South American Journal (16 février 1901): 169; ibid. (19 mars 1892): 323; ibid. (22 sept. 1928): 282; Anales de la Sociedad rural argentina (1917): 346 ; Goodwin, op. cit., 43-44 et 73; Coni, op. cit., 107-108. 
nette infériorité. En deuxième lieu, l'absence d'élévateurs de campagne a rendu impossible le système de paiement aux agriculteurs sous forme de certificats de dépôt et a entravé encore plus l'obtention d'un crédit agricole déjà hors de la portée de la plupart des fermiers pampéens.

Comment justifier l'existence d'un tel écart dans les méthodes de manutention et de mise en marché du blé canadien face au blé argentin malgré les nombreuses et importantes similitudes des deux économies frumentaires ? Pourquoi en particulier, l'Argentine ne s'est-elle jamais donné un réseau d'élévateurs complet permettant l'exportation en vrac alors que la preuve de l'efficacité et de la rentabilité de ce système était fournie depuis longtemps par le Canada et les Etats-Unis ? Pour trouver le mot de cette énigme, il faut faire appel à deux types d'explication, le premier invoquant paradoxalement la dépendance envers les marchés extérieurs dont j'ai dit plus haut qu'elle constituait un dénominateur commun des deux économies du blé et le second nécessitant un examen des sociétés respectives.

\section{4 - APPROCHE EXTERNE : LES EXIGENCES DU MARCHÉ}

L'impact de la dépendance des économies frumentaires envers l'étranger s'est exercé en sens opposé sur le Canada et sur l'Argentine. Les marchés importateurs de l'Europe ont d'abord manifesté leur influence par le biais de la structure saisonnière de la demande, jointe à l'époque de l'année où la production argentine et canadienne devenait disponible à l'exportation. Le continent européen produisait lui-même plusieurs millions de tonnes de froment mis en marché essentiellement entre les mois de juillet et décembre. Or, en plus de leur insuffisance quantitative, ces récoltes apportaient des blés mous auxquels les meuniers devaient ajouter des quantités importantes de blés durs afin d'obtenir des farines de bonne valeur panificatrice. Il se créait donc, surtout à l'automne, une très forte demande de blés durs (que l'Europe ne produisait pas) appelés "correcteurs". D'autre part, pendant les mois de janvier à juillet, c'est surtout une déficience quantitative que l'Europe cherchait à combler par ses achats outre-mer: la demande de blés demi-durs, les fillers, apparaissait alors passablement forte.

L'Argentine, pays austral, pouvait disposer de sa production à partir du mois de janvier, alors que le Canada récoltait la sienne en août-septembre. Ainsi chaque pays était soumis à une demande légèrement différente: du premier, on attendait sur- 
tout des blés demi-durs et du second surtout des blés durs. Dans la réalité, les deux vendeurs se sont conformés à l'exigence de la demande tant sur le plan de la qualité des blés que sur celui du moment de livraison. Le Canada a systématiquement exporté son blé le plus tôt possible après la récolte: au cours des années 1920 , $51 \%$ des exportations s'effectuaient entre octobre et décembre et $65 \%$ au cours des six mois suivant les moissons. Ces cargaisons se sont toujours composées de blés durs pour la presquetotalité: le Red Fife puis le Manitoba dur, et les Northern 1, 2, 3, par exemple. ${ }^{23}$ L'Argentine, d'autre part, a également concentré ses ventes à l'étranger au cours des six mois suivant les récoltes: elle exportait plus de $73 \%$ de son blé pendant cette période de l'année (1901 à 1910 et 1922 à 1931) dont 40\% au cours des trois premiers mois seulement. Il s'agissait toujours de froments à ténacité moyenne. ${ }^{24}$

Cette simple constatation est grosse d'implications de première importance. Pour réussir à exporter des blés "correcteurs", eux-mêmes répartis en plusieurs sous-catégories, le Canada devait coûte que coûte disposer d'un réseau intégral d'élévateurs; car il est virtuellement impossible de classifier avec précision des froments ensachés. Par ailleurs, la vente des blés moyens de la pampa s'accommodait somme toute assez bien du système des sacs puisque ce que les acheteurs en attendaient était davantage une "bonne moyenne" sur les grandes quantités que des qualités très spécifiques; et ainsi la nécessité des élévateurs apparaissait concrètement beaucoup moins pressante qu'en théorie.

Il est utile de noter au passage que la différence de qualité des blés argentins et canadiens n'avait rien à voir avec les techniques agricoles. En effet, les deux agricultures se rejoignaient aisément sur ce plan. Si à la fin des années 1920, l'Ouest canadien utilisait déjà largement le tracteur et le camion pendant que la pampa argentine commençait à peine à le faire, celle-ci avait fait appel aux moissonneuses-batteuses dès avant la guerre alors que celui-là y arrivait à peine à la fin des années 1920: en 1928 on dénombrait une moissonneuse-batteuse par 572 ha. cultivés en

23 Ministère du commerce du Canada, Bureau fédéral de la statistique, Report on the Grain Trade of Canada (1921-1928); MacGibbon, op. cit., 479-480, 485 et 186-188; Ministère du commerce du Canada, Annuaire du Canada, passim 1910 à 1929 .

24 República argentina, Dirección general de estadística, Anuario del comercio exterior de la República argentina (1901-1910); Coni, op. cit., 112-115 et passim; Enrique C. Urien, Geografía económica de la provincia de Bwenos Aires (Buenos Aires, 1932), 153. 
céréales en Argentine contre une par 1739 ha. en 1931 dans l'Ouest canadien. Les terres à blé s'étendaient dans les deux pays sur des surfaces très comparables: 162 ha. en 1931 en Saskatchewan-Alberta et 173 ha. dans les cinq provinces de la pampa. ${ }^{25} \mathrm{~A}$ quelques nuances près, les deux pays étaient donc également bien équipés pour l'agriculture céréalière extensive. D'autre part, la qualité différentielle des froments respectifs se manifestait par la progression constante des exportations de farine canadienne face à la stagnation des ventes de farine argentine après 1905 (voir le tableau 1). La première se composait des blés de la plus haute qualité alors que l'autre apparaissait plutôt médiocre aux clients européens. ${ }^{26}$

Il peut sembler un sophisme de tracer une relation de cause à effet entre la structure de la demande européenne de blé et l'existence ou l'inexistence d'un réseau d'élévateurs en Argentine et au Canada. A première vue, il se pourrait bien que les Canadiens aient profité de leurs installations pour exporter des froments de première qualité pendant que les Argentins dépourvus d'élévateurs, se soient contentés de vendre du blé médiocre. En tout cas, nombreux sont ceux qui ont vu le problème plutôt de cette seconde façon. Je persiste néanmoins dans mon raisonnement, en m'appuyant sur l'étude de l'évolution mensuelle des prix sur le marché de Liverpool et sur l'accumulation des coûts de rétention du blé dans les silos.

Examinons d'abord le cas argentin qui est le plus simple. C'est l'évolution mensuelle des prix internationaux qui a directement déterminé la concentration des exportations de blé argentin dans les premiers mois de l'année, telle que vue plus haut, et conséquemment, la faillite relative des efforts de mise en marché en vrac. Dans une étude minutieuse de l'importance du froment argentin sur les marchés de Liverpool, Anvers, Mannheim et Hambourg, Becker a remarqué que leur prix de février à juin de chaque année (1884 à 1902) a été plus élevé que leur moyenne annuelle dans environ $70 \%$ des cas. Timoshenko a démontré pour la période de 1893 à 1913 que sur le marché de Liverpool les blés ont obtenu en avril-mai quatre cents par

$25 \mathrm{D}$ 'après Marcelo Conti, Lo que deben conocer nuestros agricultores sobre la cosecha de trigo (Buenos Aires, 1929), passim; Aparicio, op. cit., 204 et 210; República argentina, Ministerio de agricultura, Anuario agropecuario, 1932; Septième recensement du Canada, 1931, VIII: 556, 612 et 684; Ministère du commerce du Canada, Bureau fédéral de la statistique, Handbook of Agricultural Statistics (août 1955): 7; W. A. Mackintosh, The Economic Background of Dominion-Provincial Relations (Toronto, 1964), 87.

26 MacGibbon, op. cit., 411-413. 
boisseau de plus que les blés de septembre-octobre.27 Au printemps les récoltes de l'hémisphère nord étaient déjà grevées par trois ou six mois de coûts de rétention alors que les blés argentins épargnaient le prix du stockage. En juin-juillet, la situation s'inversait au profit des exportateurs de l'hémisphère nord qui commençaient à vendre leurs récoltes alors que ceux de l'hémisphère sud avaient accumulé plusieurs mois de frais de rétention. A propos des années 1922 à 1931, Coni a exposé très clairement ce phénomène: les prix du blé à Buenos Aires diminuaient de mois en mois; le mois le plus favorable est resté janvier et le meilleur trimestre a toujours été le premier. Il n'existe aucune raison de croire qu'il n'en est pas allé exactement de même pendant toute la période de 1870 à 1930 . La croyance tant répandue d'une baisse de prix au moment de la récolte s'avère donc totalement fausse, même s'il ne s'agit que des prix bruts: d'un trimestre à l'autre entre 1922 et 1931, ceux-ci ont diminué de l'indice 100 à 98,9 à 98,3 à 97,0 . Si on considère les prix nets, l'indice dégringole encore plus vite: le 100 à 95,4 à 92,3 à $88,3 .{ }^{28}$

L'Argentine ayant tout à perdre à stocker ses froments, la construction d'un réseau d'élévateurs dans ses campagnes apparaissait donc comme un projet tout à fait hors-contexte emprunté à des pays dont la situation commerciale différait radicalement de la sienne. L'inutilité de garder son froment afin d'obtenir de meilleurs prix a découragé la mise en place des élévateurs de campagne et limité celle des silos portuaires. L'Argentine y aurait gagné les bienfaits d'une classification et d'un contrôle de la qualité; mais même cela ne présentait que des avantages restreints, puisque la différence de prix entre le blé filler et le blé "correcteur" ne justifiait certes pas l'énorme investissement nécessaire à la mise en place d'un réseau complet d'élévateurs, d'autant moins que la demande de "correcteur" se manifestait surtout en août-décembre, huit mois après la récolte dans l'hémisphère sud.

Le double phénomène de la tendance à une baisse des prix à l'automne - hausse des prix au printemps et de l'accumulation des coûts de rétention - a également conditionné l'économie canadienne du blé. L'évolution des prix aurait pu en théorie pousser le Canada, équipé qu'il était en moyens de stockage, à garder sa récolte jusqu'en janvier et à en disposer au moment de la période de hausse. Mais plusieurs facteurs poussaient à vendre

${ }^{27}$ Max Becker, Der Argentinische Weizen im Weltmarkte (Jena, 1903), passim; Timoshenko, op. cit., 26 et 99.

28 Coni, op. cit., $42,27,46$ et 48 . 
rapidement malgré tout. D'abord, selon un mécanisme analogue à celui décrit plus haut dans le cas argentin, les frais d'entreposage auraient certainement plus que compensé l'amélioration des prix puisqu'il aurait fallu garder les céréales en silos au moins pendant six mois. En outre, une politique d'attente aurait forcé le Canada à expédier, jusqu'au mois de mai, tout son froment par les ports américains, ceux du Saint-Laurent ou des Maritimes étant fermés par l'hiver ou trop éloignés. Enfin parce qu'à la seule tentative de rétention spéculative qui ait été faite à la fin des années 1920 les grosses firmes d'importation européenne ont répondu par la menace de boycotter le blé canadien au profit de l'argentin. ${ }^{29}$ Ainsi, pour maximiser ses profits, le Canada se devait lui aussi de vendre rapidement.

Bien sûr le Dominion aurait pu choisir dès le début de ne produire que du filler afin de faire l'économie de l'érection d'un réseau d'élévateurs, à la manière argentine. Mais il aurait trouvé un marché bien moins avide (aux mois de septembre-décembre) que celui dont il pouvait jouir pour ses "correcteurs", il serait entré en concurrence directe avec les exportateurs d'Europe de l'Est et il aurait par surcroît perdu l'avantage de la plusvalue de ceux-ci par rapport à celui-là. Jusqu'à un certain point, l'orientation de l'économie frumentaire du Canada a été elle aussi dictée par les consommateurs européens et la structure de leur demande.

\section{5 - APPROCHE INTERNE : LE RÔLE DES CLASSES DIRIGEANTES}

La composition et le style des sociétés canadienne et argentine ont achevé de pousser les économies frumentaires dans la direction déjà empruntée. En effet, quant à leur intérêt économique face à l'économie du blé, les groupes dominants au Canada et en Argentine des années 1880-1930 ne se situaient pas du tout sur le même plan.

$\mathrm{Au}$ Canada, le leadership social, économique et politique reposait incontestablement dans les mains de la bourgeoisie d'affaires: la banque, l'industrie et le grand commerce. C'est au sein de ce groupe social que se recrutait l'essentiel du personnel politique de premier plan, au niveau fédéral en particulier; c'est lui qui finançait les grands partis politiques; c'est lui enfin qui directement ou non influençait les décisions importantes de l'Etat central. Or, la plupart des sous-groupes de cette

29 MacGibbon, op. cit., 354. 
classe dirigeante trouvaient leur profit dans l'ouverture de l'Ouest et l'économie céréalière. Même plus, ils avaient intérêt à ce que cette percée céréalière fût la plus dynamique et la plus moderne possible de façon à maximiser leurs bénéfices.

Les autorités du Canadien Pacifique ont vite compris la relation entre la rentabilité du chemin de fer dans l'Ouest et l'ouverture d'une "frontière" agricole: dès 1884 elles ont fait construire le premier élévateur-terminus à Port Arthur. Surtout après les bonnes récoltes de 1887 au Manitoba, elles ont perçu leur avantage dans une production massive de blé dans la mesure où celle-ci augmentait le taux d'utilisation de leur matériel roulant. A partir de cette époque, elles ont ouvertement et systématiquement encouragé le développement des Prairies. Le Canadian Northern a agi de la même façon et avec la même intention quand il a fait des démarches auprès des financiers de Minneapolis pour les inciter à investir dans un réseau d'élévateurs situés le long de ses voies. ${ }^{30}$

Les banquiers et les industriels de l'Ontario avaient quant à eux des intérêts qui s'accordaient bien à ceux des compagnies de chemins de fer. Dès le début du $20 \mathrm{e}$ siècle, les premiers ont déployé un réseau de mieux en mieux organisé de succursales bancaires dans les provinces des Prairies. L'opération a réussi à un point tel qu'à la fin des années 1920 MacGibbon pouvait écrire:

The financing of the western grain crop from the time it leaves the farmer's hands until it reaches the foreign buyer is one of the largest operation that devolves annually upon the Canadian chartered banks. (...) the business furnished by the grain trade... is one of the most lucrative that (the banks) enjoy. ${ }^{31}$

Les industriels des provinces centrales, de leur côté, voyaient avec un plaisir évident se développer la population et la richesse de l'Ouest; car la prospérité par le blé signifiait l'accroissement du pouvoir d'achat d'une région non-industrialisée et l'augmentation de la demande sans poussée de la concurrence.

D'une façon plus générale, le développement de l'Ouest répondait parfaitement aux objectifs de la national policy, sousproduit typique des intérêts des milieux d'affaires de Toronto et de Montréal. Fowke l'a bien montré: "The economic imperative of the national policy ... was the establishment of a new frontier of investment opportunity which would be attached commercially

${ }^{30}$ Id ., 86-88.

31 Id., 356 et 357. 
and financially to the eastern provinces." 32 La politique tarifaire de Macdonald visait entre autres à soustraire l'Ouest à l'influence économique des Etats-Unis et à l'attacher comme une colonie interne à la bourgeoisie du Centre.

Au sein des groupes dont j'ai fait mention plus haut, il régnait d'ailleurs une assez belle harmonie qui témoignait avec une extraordinaire franchise de la communauté d'intérêt entre les grandes banques, les chemins de fer et les grosses sociétés de commerce de blé. Quelques exemples suffisent: au cours des années 1920, W. H. McWilliams présidait la Canadian Consolidated Grain Co. tout en étant un des directeurs de la Royal Bank of Canada et du National Trust Co.; J. A. Richardson participait à la direction du C.P.R. et de la Canadian Bank of Commerce tout en dominant son Eastern Terminal Elevator Co. et en jouant un rôle important comme exportateur de blé; S. T. Smith possédait sa propre compagnie d'exportation et dirigeait la Bank of Nova Scotia; et ainsi de suite..$^{33}$

La grande bourgeoisie anglo-canadienne se trouvait impliquée directement, profondément et profitablement dans l'économie du blé. On ne saurait donc se surprendre de constater que c'est elle qui, de concert avec le gouvernement fédéral, a investi l'essentiel du capital requis pour la mise en place de l'infrastructure de l'économie du blé: élévateurs, chemins de fer, etc. $\mathrm{Au}$ début de l'épopée du blé, les compagnies de chemins de fer ont même poussé le zèle jusqu'à établir leurs propres fermes expérimentales et jusqu'à fournir gratuitement des semences aux colons. $^{34}$

Ce que la grande bourgeoisie canadienne n'a pas fait ellemême elle l'a confié à son allié de toujours - d'aucuns diraient sa créature - à savoir l'Etat, surtout le fédéral. Ce dernier, en effet, s'est acquitté de sa tâche avec une diligence et un succès tout à fait remarquables. Il a d'abord joué un rôle essentiel sur le plan de la législation. Dès 1885-1889 le gouvernement d'Ottawa a commencé à réglementer le commerce des céréales et à imposer des inspections rigoureuses; de nombreuses enquêtes publiques ont amené les importantes lois canadiennes des grains de 1906, 1912 et 1925 où les règlements du commerce céréalier se sont échafaudés en un système qui faisait école à l'échelle mondiale. Les gouvernements des provinces de l'Ouest ont égale-

32 V. C. Fowke, The National Policy and the Wheat Economy (Toronto, 1957), 282.

33 MacGibbon, op. cit., passim, 322-327.

34 Id., 480. 
ment joué un rôle législatif très positif: ne citons que leurs nombreuses lois facilitant l'organisation des coopératives de producteurs et la diffusion du crédit. Au-delà de ce rôle quelque peu passif, les Etats sont intervenus dans l'économie du blé en tant qu'agents actifs. Ottawa a créé en 1919 le Canadian Wheat Board; dès 1886 il a épaulé de ses deniers les recherches agronomiques qui ont conduit, par exemple à la mise au point du blé Marquis; dès le début du siècle, il s'est mis sur les rangs des propriétaires de gros élévateurs. ${ }^{35}$ Et combien d'autres exemples de ce genre. Finalement tout concordait dans la direction de la mise sur pied d'une économie frumentaire bien organisée, moderne, efficace et rentable.

Le paysage social de l'Argentine se distinguait radicalement de celui du Canada. Là le pouvoir économique et politique appartenait non pas à une élite industrielle, financière et commerciale mais à une oligarchie de grands propriétaires terriens. C'est elle qui accaparait l'essentiel des revenus de l'économie céréalière comme de l'économie pastorale. Ferrer s'est livré à quelques calculs: "On peut estimer qu'environ $70 \%$ du réseau brut du secteur agropastoral se concentrait dans pas plus de $50 \%$ de la population active occupée dans ce secteur, c'est-à-dire, en termes nationaux, qu'environ $2 \%$ de la population recevait $20 \%$ du revenu brut du pays." 36

Cette minorité riche d'estancieros contrôlait le plus clair de la propriété du sol; en d'autres mots, elle gardait solidement le contrôle des principaux moyens de production d'un pays où l'industrie n'a commencé à percer timidement que vers 1914-1922. Détentrice du pouvoir économique, l'oligarchie argentine assurait également jusqu'en 1930 la direction politique du pays, comme l'a excellemment montré Peter Smith. ${ }^{37}$

D'autre part, l'élite ne possédait hors du secteur de la terre et de la production agropastorale que des intérêts économiques négligeables: elle ne participait pas, ou si peu, aux bénéfices du chemin de fer, des banques, des industries ou des sociétés d'exportation de céréales. Son profit se trouvait presque exclusivement dans l'enchérissement de la terre, dans le bœuf et le mouton, et dans les revenus qu'elle tirait de la location d'une partie de

35 W. T. Easterbrook et H. G. J. Aitken, Canadian Economic History (Toronto, 1967), 499 et 500; MacGibbon, op. cit., 45, 46, 62, 57-61, 66-67, 480-482, 478, 49, 384-385 et 44; Ministère du commerce du Canada, Annuaire du Canada 1912: 451, 291 et 293; 1925: 1030, et 721-722; 1916-1917: 703; 1919: 556.

36 Ferrer, op. cit., 135.

37 Peter H. Smith, Politics and Beef in Argentina (New York, 1969). 
ses terres aux fermiers céréaliers. La classe dirigeante argentine avait mis toute sa richesse et, dirais-je même, tout son honneur dans l'élevage. Le reste se faisait dans la plupart des cas sans elle et presque malgré elle. L'oligarchie agissait d'une manière qui rappelle le schéma baldwinien de l'économie de plantation. En effet, l'activité économique importante hors du secteur pastoral était surtout assurée par les étrangers. Les capitaux locaux - seuls les grands propriétaires pouvaient en disposer en quantité appréciable - ont abandonné complètement tout le grand commerce aux étrangers: les principaux exportateurs étaient les Bunge y Born, Dreyfus et assimilés, succursales de grosses sociétés européennes. A l'exception de la petite industrie, aux mains d'immigrants récents, presque tous les secteurs de production industrielle appartenaient aux Anglais ou aux Américains. Les cinq plus grandes compagnies de chemin de fer de la pampa étaient marquées "propriété britannique". Les principales banques conduisant les opérations internationales de commerce étaient également anglaises: la Bank of London and the River Plate et la British Bank of South America, par exemple. La classe dirigeante a, quant à elle, concentré ses revenus dans le secteur pastoral dont elle s'est fait une chasse-sévèrement-gardée. Elle n'a même pas participé directement à l'agriculture céréalière, second pôle de développement économique du pays: cultiver la terre était l'affaire des immigrants italiens. Scobie a bien résumé la situation: "Argentina as a nation was not concerned with the conditions of farming or of farmers". ${ }^{38}$ Et la nation, en termes politiques, c'était l'oligarchie des éleveurs.

On ne trouve donc pas en Argentine comme au Canada d'intérêt précis et direct de la part de la classe dominante dans la maximation et l'optimation de l'économie du blé, non plus qu'on perçoit de complicité entre elle et l'Etat pour faire le travail avec elle ou à sa place. L'industrie argentine des céréales s'est développée d'elle-même, au seul gré de la demande étrangère. L'Etat, certes, a légiféré pour faciliter l'accession des immigrants à la terre, pour mettre le crédit à leur portée, pour améliorer les semences ou pour rendre possible l'existence des coopératives. Mais la règle s'appliquait toujours: trop peu, trop tard. Ou encore, l'oligarchie manœuvrait de façon à détourner les lois à son seul profit. Bien plus, dans des secteurs aussi importants que la réglementation de classification du blé ou la construction d'élévateurs, l'Etat s'en est béatement remis aux bonnes volontés privées, c'est-à-dire dans ce cas aux étrangers. Ceux-ci, de leur côté, comprenant bien les déterminismes imposés à l'économie du

${ }^{38}$ Scobie, Revolution ..., op. cit., 152. 
blé de l'Argentine par la structure des marchés européens, n'ont pas bougé non plus. C'est ici qu'on voit clairement comment se rejoignaient, tout en s'appuyant sur des causalités différentes, le manque d'intérêt relatif de l'oligarchie pour l'économie du blé et la tyrannie de la dépendance envers l'extérieur pour converger en une attitude de laisser-faire.

Tout à fait divergentes quant à leurs intérêts économiques face à l'économie du blé, les classes dirigeantes canadienne et argentine ne se situaient pas non plus dans une semblable position en relation avec les groupes d'agriculteurs respectifs. Dans un cas, les positions sociales, quoique conflictuelles à de nombreux égards, s'harmonisaient sur l'essentiel, alors que dans l'autre cas elles s'entrechoquaient presque constamment. Dans cette sociologie différentielle résidait un autre élément de détermination des structures de l'économie céréalière.

La base du pouvoir des groupes dominants au Canada consistait, on le sait, dans l'industrie, la banque et le commerce. Ainsi les intérêts de la bourgeoisie n'entraient pas fondamentalement en opposition avec ceux des agriculteurs de l'Ouest dont toute l'activité se cantonnait dans l'agriculture. Quant à l'assise géographique de ce pouvoir, les deux groupes restaient bien distincts, les uns s'appuyant surtout sur le Canada central, les autres uniquement sur les provinces des Prairies. Ainsi la présence et la croissance d'une classe d'agriculteurs nombreuse, bien organisée et prospère, ne nuisaient en rien à la bourgeoisie d'affaires. Tout au contraire: le développement optimal de l'Ouest ne faisait que renforcer la position du Centre dans la mesure où, plus ou moins directement, il représentait des occasions de profits plus nombreuses pour les milieux d'affaires. En termes économiques, une distribution des revenus plutôt égalitaire favorisait une augmentation de la demande agrégée et ainsi répondait aux intérêts des bourgeois bien mieux qu'une agriculture axée socialement autour d'une oligarchie terrienne concentrant en peu de mains l'essentiel des revenus agricoles.

Dans ce contexte, on comprend mieux deux phénomènes très importants de l'histoire socio-économique de l'Ouest et essentiels au plein succès de l'économie du blé au Canada: le homestead et l'organisation économico-politique des fermiers. Mise au point en 1871-1872, la loi du homestead a été appliquée avec méthode et somme toute avec un éclatant succès malgré quelques bavures épisodiques. En plus des 25 millions d'hectares de terre vendus ou aliénés le gouvernement fédéral avait jusqu'en 1928 distribué gratuitement plus de 23 millions ha. de terre dans les provinces 
des Prairies, rendant ainsi possible l'existence d'une classe d'agriculteurs-propriétaires nombreuse et relativement homogène. ${ }^{39}$ La proportion des propriétaires parmi les agriculteurs des trois provinces des Prairies a oscillé de 1901 à 1931 entre $92 \%$ et $69 \%$; la réalité était encore plus belle si on ajoute les terres cultivées "par le propriétaire et le locataire". Au début des années 1920, on estime que près de $80 \%$ des surfaces agricoles des Prairies étaient exploitées par des agriculteurs-propriétaires. ${ }^{40}$ La politique du homestead avait donné de beaux fruits.

Maîtres de leur exploitation pour la grande majorité, les fermiers ont vite commencé à s'organiser afin de défendre leurs intérêts. Dès 1901-1903, les agriculteurs ont posé les premiers jalons de ce qui allait devenir une puissante organisation coopérative dont le but avoué était de résoudre par leurs propres forces les problèmes de manutention et d'entreposage des céréales ainsi que de faire pression sur l'Etat pour obtenir des lois régissant équitablement le commerce du blé. Avant 1910, chacune des trois provinces possédait son association de producteurs; en 1916 le United Grain Growers et la Saskatchewan Grain Growers Association démontraient déjà une vitalité étonnante et participaient directement au commerce du blé par le biais d'un réseau d'élévateurs bien à eux. A la fin des années 1920, l'organisation des fermiers culminait dans la mise sur pied des pools dont on connaît le rôle de premier plan. Au cours de la période 1924-1930, au-delà de $55 \%$ des fermiers faisaient partie des pools qui avec plus du tiers des élévateurs de campagne ont mis en marché la moitié du blé de l'Ouest vers 1926-1930.41

Les organisations de producteurs agricoles ont exercé leur puissance non seulement dans le secteur de la production et de la mise en marché du blé mais également dans le secteur politique. Bien organisés, socialement homogènes et largement majoritaires dans les Prairies, les fermiers de l'Ouest étaient en mesure de faire efficacement pression sur les gouvernements. Ils ont de fait poussé constamment sur leurs dirigeants provinciaux afin d'obtenir des politiques qui leur fussent favorables. Continuellement aussi, ils ont influencé Ottawa, directement ou non, par le seul poids politique qu'ils représentaient sur la scène fédérale. ${ }^{42}$

39 Fowke, op. cit., 60 et 284.

40 D'après: Urquhart et Buckley, op. cit., 351; Septième recensement du Canada, 1931, VIII: XIV; MacGibbon, op. cit., 456-457.

41 Ministère du commerce du Canada, Annuaire du Canada, 1925: 720721; MacGibbon, op. cit., 50-51, 69-71, 329, 344-345 et 305-306; Fowke, op. cit., 244 et 246 .

42 Fowke, op. cit., chap. 9; MacGibbon, op. cit., 48-49 et 65 . 
En somme, la classe des fermiers s'est regroupée avec succès et a travaillé elle aussi dans le sens d'une optimation de l'économie céréalière: elle surtout avait avantage à ce que la mise en marché se fît de la meilleure façon possible.

L'oligarchie argentine, pour sa part, ne pouvait pas entretenir le même type de relations avec les agriculteurs que la bourgeoisie canadienne, sous peine de perdre à plus ou moins long terme sa position de classe dominante. Pour elle, laisser les fermiers céréaliers devenir propriétaires, se constituer de puissantes organisations, accumuler des richesses et surtout jouer un rôle politique de premier plan entrait directement en contradiction avec ses intérêts profonds. Les deux groupes s'alimentaient à la même table, la terre. Même si l'un s'intéressait surtout à l'élevage et l'autre aux céréales, la base économique de leur pouvoir social, réel ou potentiel, restait identique; et fatalement ils sont entrés en conflit à propos du contrôle de cette source de richesse, c'est-à-dire à propos de la propriété du sol. Par surcroît, ce sol se trouvait géographiquement le même: l'Argentine frumentaire c'était aussi l'Argentine pastorale, c'était toujours la pampa. Ainsi, contrairement à la situation canadienne, la relation classe dominante - classe des fermiers se situait d'entrée de jeu dans un contexte hautement et ontologiquement conflictuel. L'issue de la confrontation est vite apparue: pour garder son rôle prépondérant, l'oligarchie des éleveurs a maintenu les agriculteurs-immigrants en étroite tutelle, ne leur permettant pas d'accéder à la propriété de la terre et, par conséquent, leur barrant la route du pouvoir économique et politique. Il en a résulté que les principaux intéressés à l'économie du blé n'ont jamais été en mesure d'en orienter les structures dans le sens d'une optimation des techniques de mise en marché.

L'Argentine a certes connu des plans de colonisation de style homestead. Plusieurs lois ont même été votées en ce sens entre 1853 et 1890 . De nombreux immigrants ont pu s'en prévaloir, surtout avant 1890, et acquérir des terres en toute propriété. Mais ce mouvement s'est arrêté précisément pendant les années 1890, au moment où l'exportation agropastorale commençait à devenir une affaire très intéressante. L'oligarchie a manœuvré de façon à dénaturer les lois et à s'approprier les immenses étendues encore vierges de la riche pampa. Dès 1895, les terres pampéennes avaient toutes été adjugées, non pas aux immigrantsagriculteurs mais surtout aux oligarchies traditionnelles qui souvent ne s'en servaient qu'à des fins de placement financier ou de prestige social. Quoi qu'il en soit, la politique du homestead s'avérait un lamentable échec. De son maximum de $40 \%$ en 
1898-1900, la proportion des propriétaires parmi les fermiers céréaliers a graduellement décliné jusqu'à $30 \%$ environ en 1937.43 C'est donc dire que la production frumentaire s'est appuyée de plus en plus sur des fermiers locataires et itinérants. En 1894, les aristocrates Anales de la Sociedad rural argentina admettaient elles-mêmes que les spéculateurs avaient soustrait la terre aux agriculteurs. En 1898, on pouvait lire ailleurs:

Les lois agraires de la Province de Buenos Aires ... ont été ... faites pour favoriser les intérêts des propriétaires terriers eux-mêmes ... Le triomphe du conservatisme des grands domaines était sanctionné une fois de plus par l'usage qu'ils firent des termes de la loi. ${ }^{44}$

L'aristocratie terrienne restait décidément régnante et l'agriculteur avait perdu dès la fin des années 1880 la bataille de l'accessibilité à la propriété rurale.

Dans ces conditions, il n'est pas étonnant de constater l'échec à peu près total des tentatives d'organisation des fermiers avant 1930. A peine existait-il quelques petites coopératives de consommation ou d'assurances qui ont péréclité à la première crise..$^{45}$ Absolument rien de comparable aux associations de fermiers du Canada. L'isolement, l'insécurité, l'instabilité et par-dessus tout la condition de locataire des agriculteurs ont contrecarré toute tentative d'organisation solide.

De la même façon qu'elle s'était réservé la propriété terrienne en utilisant à ses seules fins les lois de homestead, l'oligarchie a détourné à son bénéfice les crédits financiers prévus pour les fermiers. La Banco hipotecario nacional et la Banco de la Nación n'ont délié leur bourse qu'au profit de ceux qui offraient des garanties, c'est-à-dire les grands propriétaires.

Enfin, l'oligarchie n'a pas permis aux agriculteurs céréaliers d'accéder au pouvoir politique. D'ailleurs, ceux-ci étaient à ce

43 Emilio Lahitte, Cosecha del año 1898/99 (Buenos Aires, 1899), et Cosecha del año 1899/1900 (Buenos Aires, 1900), passim; República argentina, Tercer censo nacional (1914) (Buenos Aires, 1916-1917) ; id., Censo nacional agropecuario (1937) (Buenos Aires, 1939-1940); Carl C. Taylor, Rural Life in Argentina (Bâton Rouge, 1948), 194.

44 Anales de la Sociedad rural argentina (1894): 254; D. Francisco Segui, Investigación parlamentaria sobre agricultura, cité par Ricardo J. Huergo, Investigación agricola (Buenos Aires, 1904), 94.

45 República argentina, Tercer censo..., op. cit., 10: 143, 151 et passim; Scobie, Revolution..., op. cit., 14; Anales de la Sociedad rural argentina (1918): 390; Domingo Borea, Tratado de cooperacion (Buenos Aires, 1927), 621-625 et 276; República argentina, Ministerio de agricultura, Informe..., op. cit., 270 et 43 ; Coni, op. cit., 90 . 
point inorganisés et faibles que cette dernière opération s'est déroulée sans difficulté. Les agriculteurs n'ont jamais reçu l'attention spécifique et soutenue d'un parti politique déterminé. Les conservateurs, les radicaux et les socialistes se sont intéressés aux grands propriétaires et aux citadins, jamais aux agriculteurs; et personne ne les a représentés en tant que groupe auprès des gouvernements central et provinciaux. Ils étaient politiquement inexistants.

La classe dirigeante argentine pour sa part a très souvent abusé de sa prépondérance et a mené les affaires du pays comme son entreprise propre. A tous les niveaux de la politique, les gouvernements ont nommé leurs propres successeurs, ouvertement jusqu'à 1916 et d'une manière plus discrète mais non moins efficace après. On a tenu peu de compte du vote populaire que les hommes en place manipulaient sans scrupule, surtout dans les régions éloignées de Buenos Aires. On s'est partagé allègrement les postes intéressants, en tombant souvent dans le népotisme le plus grossier. Marginale, inorganisée et sans voix politique, la classe des fermiers ne disposait d'aucun moyen d'orienter les structures de l'économie du blé dans une direction autre que celle qu'imposait le jeu brutal de la demande extérieure. Les estancieros, quant à eux, se sont satisfaits de cette situation plutôt que de remettre en cause leur statut social de grands éleveurs et leur puissance économico-politique.

\section{6 - SOMMAIRE}

Un rapide bilan de cette étude permet de replacer les principales pièces du dossier dans leur éclairage mutuel. Entre 1880 et 1930, l'Argentine et le Canada ont mis sur pied des économies du blé qui s'apparentaient à de nombreux points de vue, tant par leur ampleur quantitative que par leur dépendance à l'égard de l'étranger demandeur de céréales. Pourtant l'un a réussi à bâtir un système de mise en marché qui a fait école à l'échelle mondiale pendant que l'autre se contentait sous ce rapport d'une bonne médiocrité. De cette divergence fondamentale, il faut rendre responsables les structures mêmes des marchés consommateurs de blé qui ont poussé l'un et l'autre dans des directions différentes; il importe également de constater qu'à l'intérieur de chaque pays, le jeu des forces sociales a maintenu dans la même direction les tendances déjà amorcées. Dans le cas canadien, la demande a incité à réaliser un système de commercialisation en vrac pendant que la bourgeoisie y trouvait son profit et laissait 
travailler les agriculteurs dans le même sens. En Argentine, la nature même du marché extérieur n'encourageait pas un système commercial de ce genre alors que l'oligarchie au pouvoir n'y trouvait pas son compte et qu'elle a inhibé les velléités des fermiers à essayer de corriger la situation.

\section{SOURCES DES GRAPHIQUES}

46 República argentina, Dirección general de estadística, El comercio exterior argentino en 1936 y 1935 (Buenos Aires, 1937), 105-107; República argentina, Ministerio de agricultura, Anuario agropecuario, 1932: 17; Aparicio, op. cit., 379; Scobie, Revolution..., op. cit., 170.

47 Urquhart et Buckley, op. cit., 23, 363-364, 528 et 532. 